\title{
Critical care echocardiography in prone position patients during COVID-19 pandemic: a feasibility study
}

\author{
Diego Ugalde ${ }^{1}$ (1) - Juan Nicolas Medel ${ }^{1} \cdot$ Pablo Mercado $^{2} \cdot$ Ronald Pairumani $^{3}$ - Daniela Eisen ${ }^{1} \cdot$ Edward Petruska $^{3}$.

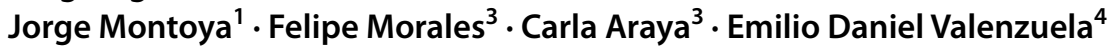

Received: 5 August 2021 / Accepted: 1 January 2022 / Published online: 28 February 2022

(c) Società Italiana di Ultrasonologia in Medicina e Biologia (SIUMB) 2022

\begin{abstract}
Purpose Critical care echocardiography is a fundamental tool in the hemodynamic evaluation of critically ill patients and prone position ventilation might limit its application. We aim to evaluate the feasibility of transthoracic echocardiography to assess different measurements performed in prone vs supine position in patients during COVID-19 pandemic to answer our research question: What is the feasibility of classic echocardiographic measurements in COVID-19 patients in prone position ventilation?

Methods Patients with covid-19 admitted to ICUs in four academic hospitals with respiratory failure and on mechanical ventilation were evaluated with critical care echocardiography. The first ultrasound assessment was compared between prone and supine patients recording feasibility of several echocardiographic measurements, using Fisher's exact test complementing with Crombach's Alpha.

Results 139 patients were included. Sixty-eight (49\%) were evaluated in prone position and seventy one (51\%) in supine position. Most variables were highly feasible, left ventricular volumes and ejection fraction were more possible to obtain in prone position, while cardiac output was in supine position. Tricuspid regurgitation was the least feasible overall measurement.

Conclusion Prone position ultrasound achieved a high feasibility of measurements compared with supine ultrasound in critically ill patients with COVID-19 respiratory failure and on mechanical ventilation.

Registration Post hoc analysis of Echo-COVID study (NTC04628195, registered November 13, 2020, retrospectively registered).
\end{abstract}

Keywords COVID-19 $\cdot$ Respiratory insufficiency $\cdot$ Echocardiography $\cdot$ Prone position $\cdot$ Mechanical ventilation $\cdot$ Critically ill

Diego Ugalde

diegougaldecastillo@gmail.com

1 Departamento de Medicina, Hospital Clínico Universidad de Chile, Unidad de Pacientes Críticos, Dr Carlos Lorca Tobar 999, Santiago, Chile

2 Departamento de Paciente Crítico, Clínica Alemana de Santiago, Facultad de Medicina Clínica Alemana, Universidad del Desarrollo, Santiago, Chile

3 Hospital Barros Luco Trudeau, Unidad de Cuidados Intensivos, Santiago, Chile

4 Departamento de Medicina Intensiva, Facultad de Medicina, Pontificia Universidad Católica de Chile, Santiago, Chile

\section{Background}

Critical care echocardiography (CCE) is nowadays recommended as the first line evaluation technique in hemodynamically unstable patients, particularly to diagnose type of shock and guide the hemodynamic resuscitation; CCE allows the operator to perform qualitative assessments, quantitative measurements and real time follow-up [1,2].

Covid-19 pandemic has imposed a work overload on healthcare staff in intensive care units (ICUs) with patients with severe respiratory failure and high mortality rates [3-6] many of them requiring prone position as recommended in acute respiratory distress syndrome (ARDS) guidelines [7]. It is known that during ARDS the right ventricle (RV) can be compromised and its evaluation becomes important to adjust ventilation parameters, in particular for detection of 
acute cor pulmonale pattern (ACP), considering its association with poor outcomes and its more frequent presentation in the most severe ARDS patients [8-11].

In this context, with higher workload and increased number of patients, transthoracic CCE in prone position might be a valuable tool for quicker and simpler evaluation in comparison to the transesophageal approach, and since the technique was reported [12] it has been increasingly described in recent series with new updates and variations, particularly during the current pandemic [13-15]. Thus, our research question: What is the feasibility of classic echocardiographic measurements in COVID-19 patients in prone position ventilation?

Our objective was to describe the feasibility of different echocardiographic measurements performed in prone vs supine position in COVID-19 patients during the current pandemic season in four academic centers in Chile.

\section{Material and methods}

This study is a post hoc analysis of Echo-COVID study (NTC04628195). From March to June 2020, COVID-19 patients admitted to ICUs in four academic hospitals in Chile were evaluated with repeated cardiac ultrasound. Patients were included if they were admitted to ICU and were on mechanical ventilation with confirmed positive COVID-19, either by polymerase chain reaction test or a positive Computer Tomography with serology; and respiratory failure was attributed to covid-19. We excluded patients with no ultrasound window.

From this cohort we evaluated the first cardiac ultrasound performed identifying those done in prone or supine position and evaluating the feasibility of measurements.

The prone position echocardiography was performed in the left swimmer position as previously described [12], aiming to obtain apical view and related measurements. Supine views were performed without modifications to patient position. All ultrasounds were performed by operators with at least two years of critical care ultrasound experience. Only one operator evaluated each patient.

We included primary measurements, usually used alone or in calculations that allow characterization of left and right ventricular systolic and diastolic function and orientation about fluid status. We included the feasibility of qualitative assessment of septal movement considering the relevance of right heart function in COVID-19 and cor pulmonale. In addition, the feasibility of calculated relevant variables was included. The list of variables evaluated were: Left ventricle (LV) end diastolic and end systolic volume in four chambers view, MAPSE, TAPSE, right ventricle (RV) end diastolic area in four chambers view, LV end diastolic area in four chambers view, trans-tricuspideal gradient, septal morphology, mitral A wave, Mitral E wave, tissue doppler (TD) s' and e' mitral waves, TD s' tricuspid wave, LVOT (left ventricle outflow tract) velocity time integral (VTI) and inferior vena cava (IVC) maximum and minimum diameter. LVOT diameter is obtained in the parasternal window and feasibility was not recorded given its intrinsic impossibility in prone position. The following "integrated" variables feasibilities were recorded and compared: Cardiac output, $\mathrm{LV}$ ejection fraction, acute cor pulmonale (ACP) detection, diastolic function and fluid responsiveness using IVC despite known limitations. Feasibility was dichotomic, either feasible or not feasible. Any measurement that was not recorded by the operator was defined as not feasible.

Echocardiographic measurements were obtained with a Vivid i echocardiography system (GE Medical Systems, Milwaukee, WI, USA), Philips CX 50 (Philips Healthcare, DA Best, The Netherlands), Mindray M9 (Bio-Medical Electronics Co., Shenzhen, China), and Sonosite M-Turbo (FUJIFILM Sonosite, Inc. Bothell, WA, USA), as used in each center. Invasive measurements were not available since pulmonary artery catheter use is uncommon in our centers.

Ethical approval was granted from local ethics boards of each center (ID: 20042,002).

The proportion of feasible measurements was obtained, described and then the proportion of each one was compared using Fisher's exact test in SPSS 20 between supine and prone group, $p$ value under 0.05 was considered statistically significant. We evaluated Cronbach's $\alpha$ for 2D (LVOT diameter, LV end diastolic and systolic volumes, LV ejection fraction, MAPSE, TAPSE, RV and LV end diastolic areas, IVC minimum and maximum diameters) and doppler (trans-tricuspideal gradient, mitral A and E waves, s' and e' mitral waves, s' tricuspid wave, LVOT VTI) measurements in supine and prone position groups.

\section{Results}

One hundred sixty patients were evaluated for 4 months. 21 patients were excluded because no ultrasound window could be obtained, twelve (14.46\%) in supine position and nine $(11,69 \%)$ in prone position with no significant difference. Finally, 139 patients were included for analysis. Sixty-eight patients (49\%) were evaluated in prone position and seventy-one (51\%) in supine position. Clinical and demographic characteristics are summarized in Table 1, no difference between groups was observed, the group overall included mostly males around 55 years old with a week of COVID19 symptoms before admission and 9-10 before ventilation.

The feasibility of different echocardiographic measurements, calculation and integrated variables are shown in Table 2, for all patients in both groups with the appropriate statistical comparisons. Most variables showed high 
Table 1 Demography and clinical characteristics
Table 2 Absolute frequency and proportion of feasibility of echocardiographic variables

\begin{tabular}{lllll}
\hline Variable & All patients & Prone & Supine & $p$ value* \\
\hline Sex, male (\%) & $99(71.22 \%)$ & $48(70.59 \%)$ & $51(71.83)$ & 0.51 \\
Age, years + & $57.21+11.61$ & $55.76+11.41$ & $58.58+11.70$ & 0.15 \\
Height, m+ & $1.67+0.09$ & $1.67+0.09$ & $1.68+0.10$ & 0.699 \\
Weight, Kg + & $86.53+17.99$ & $87.69+18.36$ & $85.46+17.70$ & 0.479 \\
SOFA score at ICU Admission + & $6.29+2.63$ & $6.55+2.91$ & $6.00+2.27$ & 0.261 \\
APACHE II + & $14.47+6.29$ & $15.57+6.63$ & $13.29+5.74$ & 0.50 \\
Day of symptoms before Hospital admission* & $7[5-9.25]$ & $7[4-8]$ & $7[5-10]$ & 0.363 \\
Hospital length of stay before ICU admission* & $1[0-3]$ & $1[0-5]$ & $1[0-3]$ & 0.151 \\
Days of symptoms before MV* & $9[7-13]$ & $9[6.75-13]$ & $10[7-13]$ & 0.659 \\
\hline
\end{tabular}

$I C U$ intensive care unit, $M V$ mechanical ventilation

*Median and IQR, Mann Whitney test

+ Mean and standard deviation, $T$-test

\begin{tabular}{lllll}
\hline Evaluated variable & All patients $(n=139)$ & Prone $(n=68)$ & Supine $(n=71)$ & $p$ value* \\
\hline Direct measurements & Absolut frequency $($ proportion $\%)$ & & \\
LV end diastolic volume & $124(89.21 \%)$ & $65(95.59 \%)$ & $59(83.10 \%)$ & $\mathbf{0 . 0 1 6}$ \\
LV end systolic volume & $124(89.21 \%)$ & $65(95.59 \%)$ & $59(83.10 \%)$ & $\mathbf{0 . 0 1 6}$ \\
MAPSE & $130(93.53 \%)$ & $64(94.12 \%)$ & $66(92.96 \%)$ & 0.527 \\
TAPSE & $137(98.56 \%)$ & $67(98.53 \%)$ & $70(98.59 \%)$ & 0.741 \\
RV end diastolic area & $135(97.12 \%)$ & $67(98.53 \%)$ & $68(95.77 \%)$ & 0.326 \\
LV end diastolic area & $134(96,40 \%)$ & $67(98,53 \%)$ & $67(94,34 \%)$ & 0.197 \\
TR gradient & $59(42,45 \%)$ & $32(47,06 \%)$ & $27(38,03 \%)$ & 0.183 \\
Septum morphology & $120(86.33 \%)$ & $56(82.35 \%)$ & $64(90.14 \%)$ & 0.138 \\
Mitral E wave & $137(98.56 \%)$ & $67(98.53 \%)$ & $70(98.59 \%)$ & 0.741 \\
Mitral A wave & $132(94.96 \%)$ & $67(98.53 \%)$ & $68(95.77 \%)$ & 0.476 \\
Mitral S' wave & $132(94.96 \%)$ & $65(95.59 \%)$ & $67(94.34 \%)$ & 0.524 \\
Mitral e' wave & $133(95.68 \%)$ & $65(95.59 \%)$ & $68(95.77 \%)$ & 0.639 \\
Tricuspid S' wave & $131(94.24 \%)$ & $64(94.12 \%)$ & $67(94.34 \%)$ & 0.616 \\
LVOT VTI & $134(96.40 \%)$ & $65(95.59 \%)$ & $69(97.18 \%)$ & 0.479 \\
Maximum IVC diameter & $112(80.58 \%)$ & $52(76.47 \%)$ & $60(84.51 \%)$ & 0.163 \\
Minimum IVC diameter & $112(80.58 \%)$ & $52(76.47 \%)$ & $60(84.51 \%)$ & 0.163 \\
Integrated and calculated variables & & & & $<$ \\
Cardiac Output & $110(79.14 \%)$ & $45(66.18 \%)$ & $65(91.55 \%)$ & $<\mathbf{0 . 0 0 1}$ \\
Ejection fraction & $124(89.21 \%)$ & $65(95.59 \%)$ & $59(83.10 \%)$ & $\mathbf{0 . 0 1 6}$ \\
ACP pattern evaluation & $134(96.40 \%)$ & $67(98.53 \%)$ & $67(94.34 \%)$ & 0.197 \\
Diastolic function evaluation & $127(91.37 \%)$ & $62(91.18 \%)$ & $65(91.55 \%)$ & 0.587 \\
Fluid responsiveness & $112(80.58 \%)$ & $52(76.47 \%)$ & $60(84.51 \%)$ & 0.163 \\
\hline
\end{tabular}

$L V$ left ventricle, MAPSE Mitral annular plane systolic excursion, TAPSE tricuspid annular plane systolic excursion, $R V$ right ventricle, $T R$ tricuspid regurgitation, $L V O T$ left ventricle outflow tract, $V T I$ velocity time integral, IVC inferior vena cava, $A C P$ acute cor pulmonale

*One sided Fisher's exact test

In bold letters those $<0.05$ feasibility with no differences between prone and supine patients with a feasibility over $80 \%$ in most of them. However, there were statistically significant differences in left ventricular volumes and ejection fraction evaluation in four chambers, favoring prone position $(96 \%$ vs $83 \%$, respectively), and in cardiac output calculation favoring supine position (92\% vs 66\%). The measurement with the lowest feasibility was tricuspid regurgitation with no differences between the two groups ( $42 \%$ overall). 
Cronbach's $\alpha$ for 2D measurements was 0.68 in supine and 0.64 in prone position, $\alpha$ for doppler measurements was 0.47 in supine and 0.41 in prone position.

\section{Discussion}

Our results show that $\mathrm{CCE}$ in prone ventilation has a high feasibility for many of the fundamental measurements and observations in "real-life" complex patients, particularly in the acute and most critical stage of COVID-19 respiratory failure when compared to similar patients in supine position; but some variables showed significant differences. We observed that cardiac output calculation was less feasible for prone position patients, considering that left ventricle outflow tract was equally obtained, the limitation in prone position was measuring the outflow tract diameter, which requires a long parasternal axis that is not possible in prone position ventilation. However, obtaining the outflow diameter in the patient just before being turned prone would allow the calculation of the cardiac output, as some of our team members did.

In contrast, left ventricle volumes and ejection fraction were more feasible in prone position, this is possible because of the improved apical view considering the favourable position of the heart against the chest wall in swimmer's prone position.

As for the other variables no difference was shown. We obtained a high overall feasibility considering that critical care patients might not present adequate windows in up to $40 \%$ in expert operator hands $[16,17]$. Since prone position ultrasound is focused on the apical view, a good result was to be expected in aortic and mitral flow evaluations, chamber and ejection estimation and tissue Doppler measurements. It is noteworthy that the most critical measurements such as LVOT VTI were obtained in more than $90 \%$ of cases, which is relevant to perform dynamic manoeuvres for better characterization of the hemodynamic condition of each patient and fluid or inotrope tailoring if required [16-19]. Variables that were harder to obtain in both groups include IVC diameters (around 80\%) and particularly tricuspid gradient, possibly because of window limitations or small gradient regurgitations that did not allowed appropriate tracing.

Another relevant aspect is the high proportion of RV evaluation to discern whether ACP was present, this requires appropriate septal evaluation and RV/LV area measurements, and a high proportion of achievement is paramount in ARDS particularly in severe cases requiring prone position [8-11]. Considering the good feasibilities, the requirement of a transoesophageal examination [20] for undetermined cases should be reduced, diminishing resource utilization, particularly in the present pandemic setting.
The obtained Cronbach's $\alpha$ suggest a better consistency for 2D measurements than doppler derived variables.

This study has several limitations, it was performed only in academic centers having operators with prone CCE experience, thus, feasibility could be worse in unexperienced hands and these results might not represent results in nonacademic hospitals. However, in our opinion, prone position ultrasound is very similar to supine position and considering that the heart becomes in close contact with the chest wall [21] the views might be even easier to obtain even for a basically trained operator; in addition, we think that prone position ultrasound should be considered as part of conventional training and practiced whenever it is possible for the trainee considering the valuable information in can provide.

\section{Conclusion}

Cardiac ultrasound in prone position allows hemodynamic evaluation in COVID-19 patients comparable to traditional supine position ultrasound with better feasibility for LV volumes and ejection fraction and worse for cardiac output.

Author contributions EDV and JNM planned this analysis, all authors performed ultrasound on patients and obtained primary data, DU and EDV performed statistical analysis, DU wrote the first draft, DU, PM, JNM and EDV refined the final manuscript. All authors approved the final document.

Funding No funding was received.

Availability of data and material The datasets used and/or analysed during the current study are available from the corresponding author on reasonable request.

Code availability Not applicable.

\section{Declarations}

Conflicts of interest/Competing interests The authors declare that they have no competing interests.

Ethics approval Ethical approval was granted from local ethics boards of each center (ID: 200422002)

Consent to participate Consent was waived given the observational nature of the study.

Consent for publication Not applicable. The manuscript does not contain personal data.

\section{References}

1. Cecconi M, De Backer D, Antonelli M, Beale R, Bakker J, Hofer C, Jaeschke R, Mebazaa A, Pinsky MR, Teboul JL, Vincent JL, 
Rhodes A (2014) Consensus on circulatory shock and hemodynamic monitoring. Task force of the European Society of Intensive Care Medicine. Intensive Care Med 40:1795-1815. https://doi. org/10.1007/s00134-014-3525-z

2. Vieillard-Baron A, Millington SJ, Sanfilippo F, Chew M, DiazGomez J, McLean A, Pinsky MR, Pulido J, Mayo P, Fletcher N (2019) A decade of progress in critical care echocardiography: a narrative review. Intensive Care Med 45:770-788. https://doi.org/ 10.1007/s00134-019-05604-2

3. Grasselli G, Zangrillo A, Zanella A, Antonelli M, Cabrini L, Castelli A, Cereda D, Coluccello A, Foti G, Fumagalli R, Iotti G, Latronico N, Lorini L, Merler S, Natalini G, Piatti A, Ranieri MV, Scandroglio AM, Storti E, Cecconi M, Pesenti A, COVID19 Lombardy ICU Network (2020) Baseline characteristics and outcomes of 1591 patients infected with SARS-CoV-2 admitted to ICUs of the Lombardy Region. Italy. JAMA. 323:1574-1581. https://doi.org/10.1001/jama.2020.5394

4. Arentz M, Yim E, Klaff L, Lokhandwala S, Riedo FX, Chong M, Lee M (2020) Characteristics and outcomes of 21 critically ill patients With COVID-19 in Washington State. JAMA 323:1612 1614. https://doi.org/10.1001/jama.2020.4326

5. Myers LC, Parodi SM, Escobar GJ, Liu VX (2020) Characteristics of hospitalized adults With COVID-19 in an Integrated Health Care System in California. JAMA 323:2195-2198. https://doi. org/10.1001/jama.2020.7202

6. Wang Y, Lu X, Li Y, Chen H, Chen T, Su N, Huang F, Zhou J, Zhang B, Yan F, Wang J (2020) Clinical course and outcomes of 344 intensive care patients with COVID-19. Am J Respir Crit Care Med 201:1430-1434. https://doi.org/10.1164/rccm. 202003-0736LE

7. Papazian L, Aubron C, Brochard L, Chiche JD, Combes A, Dreyfuss D, Forel JM, Guérin C, Jaber S, Mekontso-Dessap A, Mercat A, Richard JC, Roux D, Vieillard-Baron A, Faure H (2019) Formal guidelines: management of acute respiratory distress syndrome. Ann Intensive Care 9:69. https://doi.org/10.1186/ s13613-019-0540-9

8. Jardin F, Dubourg O, Bourdarias JP (1997) Echocardiographic pattern of acute cor pulmonale. Chest 111:209-217. https://doi. org/10.1378/chest.111.1.209

9. Mekontso Dessap A, Boissier F, Charron C, Bégot E, Repessé X, Legras A, Brun-Buisson C, Vignon P, Vieillard-Baron A (2016) Acute cor pulmonale during protective ventilation for acute respiratory distress syndrome: prevalence, predictors, and clinical impact. Intensive Care Med 42:862-870. https://doi.org/10.1007/ s00134-015-4141-2

10. Vieillard-Baron A, Prin S, Chergui K, Dubourg O, Jardin F (2002) Echo-Doppler demonstration of acute cor pulmonale at the bedside in the medical intensive care unit. Am J Respir Crit Care Med 166:1310-1319. https://doi.org/10.1164/rccm.200202-146CC

11. See KC, Ng J, Siow WT, Ong V, Phua J (2017) Frequency and prognostic impact of basic critical care echocardiography abnormalities in patients with acute respiratory distress syndrome. Ann Intensive Care 7:120. https://doi.org/10.1186/s13613-017-0343-9
12. Ugalde D, Medel JN, Romero C, Cornejo R (2018) Transthoracic cardiac ultrasound in prone position: a technique variation description. Intensive Care Med 44:986-987. https://doi.org/10. 1007/s00134-018-5049-4

13. Gibson LE, Di Fenza R, Berra L, Bittner EA, Chang MG (2020) Transthoracic echocardiography in prone patients with acute respiratory distress syndrome: a feasibility study. Crit Care Explor 2:e0179. https://doi.org/10.1097/CCE.0000000000000179

14. Rali AS, Trevino S, Yang E, Herlihy JP, Diaz-Gomez J (2020) Cardiopulmonary ultrasonography for severe coronavirus disease 2019 patients in prone position. Card Fail Rev 6:e12. https://doi. org/10.15420/cfr.2020.12

15. Giustiniano E, Padua E, Negri K, Bragato RM, Cecconi M (2020) Echocardiography during prone-position mechanical ventilation in patients with COVID-19: a proposal for a new approach. J Am Soc Echocardiogr 33:905-906. https://doi.org/10.1016/j.echo.2020.04. 027

16. Jozwiak M, Depret F, Teboul JL, Alphonsine JE, Lai C, Richard C, Monnet X (2017) Predicting fluid responsiveness in critically ill patients by using combined end-expiratory and end-inspiratory occlusions with echocardiography. Crit Care Med 45:e1131e1138. https://doi.org/10.1097/CCM.0000000000002704

17. Maizel J, Salhi A, Tribouilloy C, Massy ZA, Choukroun G, Slama M (2013) The subxiphoid view cannot replace the apical view for transthoracic echocardiographic assessment of hemodynamic status. Crit Care 17:R186. https://doi.org/10.1186/cc12869

18. Muller L, Toumi M, Bousquet PJ, Riu-Poulenc B, Louart G, Candela D, Zoric L, Suehs C, de La Coussaye JE, Molinari N, Lefrant JY, AzuRéa Group (2011) An increase in aortic blood flow after an infusion of $100 \mathrm{ml}$ colloid over 1 minute can predict fluid responsiveness: the mini-fluid challenge study. Anesthesiology 115:541-547. https://doi.org/10.1097/ALN.0b013e318229a500

19. Wu Y, Zhou S, Zhou Z, Liu B (2014) A 10-second fluid challenge guided by transthoracic echocardiography can predict fluid responsiveness. Crit Care 18:R108. https://doi.org/10.1186/cc138 91

20. Mekontso Dessap A, Proost O, Boissier F, Louis B, Roche Campo F, Brochard L (2011) Transesophageal echocardiography in prone position during severe acute respiratory distress syndrome. Intensive Care Med 37(3):430-434. https://doi.org/10.1007/ s00134-010-2114-z

21. Albert RK, Hubmayr RD (2000) The prone position eliminates compression of the lungs by the heart. Am J Respir Crit Care Med 161:1660-1665. https://doi.org/10.1164/ajrccm.161.5.9901037

Publisher's Note Springer Nature remains neutral with regard to jurisdictional claims in published maps and institutional affiliations. 